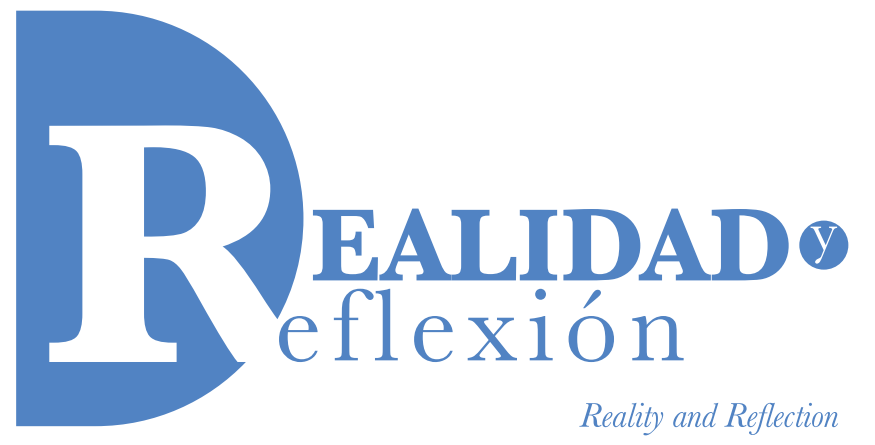

ISSN 1992-6510

e-ISSN 2520-9299

Año 18, N 47, San Salvador, El Salvador, Centroamérica. Revista Semestral Enero-Junio 2018

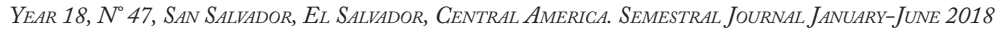

\title{
La gestión represiva de la violencia en el año 2015 en El Salvador
}

\section{The repressive management of the violence in the year 2015 in El Salvador}

\author{
Nohel M. Reyes \\ Licenciado en Relaciones Internacionales por la Universidad Francisco Gavidia \\ Estudios sobre Gobierno e Historia de los EEUU por la Universidad de Arizona \\ Estudiante del Máster en Seguridad Pública, Ciudadanía y DDHH por la Universidad del Estado del Amazonas \\ Recibido: 1 de febrero de 2018 \\ Aprobado: 22 de mayo de 2018 \\ DOI: http://dx.doi.org/10.5377/ryr.v0i47.6279
}

\section{RESUMEN}

En el año 2015 se registró en El Salvador una tasa de 103 homicidios intencionales por cada 100,000 habitantes, posicionándose como el país más violento de América Latina. En este contexto, el objetivo del presente artículo es analizar la estrategia de represión implementada por las organizaciones públicas para combatir la violencia criminal. Para esto, fue realizado un levantamiento de datos cualitativos y cuantitativos a través de fuentes secundarias, entre las cuales destacan trabajos de periodismo investigativo. Identificando que las agencias cometidas por el Ejecutivo Salvadoreño, así como por dos organizaciones centrales dentro del sistema de justicia penal, la Sala de lo Constitucional de la Corte Suprema de Justicia y la Policía Nacional Civil, parecen apuntar a una misma estrategia.

Palabras clave: gestión represión, fuerza excesiva, violencia criminal, organizaciones públicas, pandillas.

\section{ABSTRACT}

The year 2015 meant for El Salvador an intentional homicide rate of 103 per 100,000 inhabitants, positioning it has the most violent country in Latin American. In this context, the objective of the present article is to analyze the strategy of repression implemented by the public organizations to fight the criminal violence. For this, it was done a lifting of qualitative and quantitate data trough secondary resources, in which stands ups the work of research journalism. Identifying that the agencies committed by the Salvadorian Executive, as well as two central organizations inside the penal justice system, the Sala de lo Constitucional of the Corte Suprema de Justicia and the Policia Nacional Civil, appear to aim in the same strategy.

Keywords: repressive management, excessive force, criminal violence, public organizations, gangs. 


\section{Introducción}

La medición de la violencia es un desafío y representa un problema complejo. En líneas generales, existen dos fuentes de datos que son las más reconocidas y utilizadas: los registros administrativos y las encuestas de victimización, cada una de ellas con sus ventajas y desventajas.

Cuando se habla de los registros administrativos se puede tener acceso a largos periodos históricos y una desagregación territorial amplia sobre los datos que investigamos. Sin embargo, existe una desventaja significativa, la cifra oscura detrás de los datos oficiales puede ser alta debido a la falta de confianza de la población en las instituciones que colectas los datos de manera oficiosa (PNUD, 2013, p. 42).

Por otro lado, las encuestas de victimización pueden recolectar datos sobre el sentir de la población no solamente frente al actuar criminal, sino también frente al actuar de las organizaciones destinadas a la seguridad pública y ciudadana. Las desventajas que se pueden encontrar en estos instrumentos son su eventualidad y costo, no siempre se cuenta con una encuesta actualizada para consultar y en pocas situaciones se tiene los recursos necesarios para llevar a cabo un trabajo de este tipo (PNUD, 2013, p. 42).

En este contexto de limitaciones, una herramienta viable al momento de medir la violencia es el índice de homicidios para cada 100,000 habitantes, ${ }^{1}$ entre otras razones porque

\footnotetext{
1 De aquí en adelante se abreviará como IH para cada $100 \mathrm{~K}$.

2 Para más información sobre La Tregua, consultar Van der Borg, Chris y Savenije, Wim (28 de abril de 2017). Las lecciones de la Tregua. E1

Faro, San Salvador. Recuperado de: https://goo.gl/1hRvjq.
}

es más difícil ocultar el delito cometido por las pruebas físicas que genera, por el registro histórico creado debido al trabajo administrativo de las organizaciones públicas y porque no se necesita invertir recursos significativos en la colecta de los datos.

Según la Organización Mundial de la Salud (OMS), cuando un país tiene más de 10 homicidios para cada 100,000 puede declararse epidemia de salud (PNUD, 2013, p. 46). Bajo este parámetro, El Salvador nunca tuvo un desempeño deseable, según los datos sistematizados por parte del periódico digital E1 Faro, obtenidos de fuentes administrativas de la Policía Nacional Civil (PNC) y del Instituto de Medicina Legal (IML), el promedio del $\mathrm{IH}$ para cada 100,000, desde el año 2000 hasta el 2004, fue de 52.73 (Valencia, 19 de agosto de 2015).

A pesar de la tendencia anterior, el año 2015 presentó una anomalía estadística importante, la cual se inició con la nueva estrategia del Ejecutivo y que obligó las agencias de dos organizaciones públicas centrales del sistema de justicia penal. Para el 5 de enero de 2015, después de 7 meses de haber asumido el cargo, el presidente de la República de El Salvador, Salvador Sánchez Cerén, asistió a una reunión con la alta dirigencia de la PNC en la que, a puerta cerrada, se decidió finalizar definitivamente con la estrategia en materia de seguridad pública iniciada en marzo de 2012 conocida popularmente como La Tregua (Valencia, 13 de abril de 2015). ${ }^{2}$

A pesar de la tendencia anterior, el año 2015 presentó una anomalía estadística importante, la cual se inició con la nueva estrategia del Ejecutivo 
y que obligó las agencias de dos organizaciones públicas centrales del sistema de justicia penal. Para el 5 de enero de 2015, después de 7 meses de haber asumido el cargo, el presidente de la República de El Salvador, Salvador Sánchez Cerén, asistió a una reunión con la alta dirigencia de la PNC en la que, a puerta cerrada, se decidió finalizar definitivamente con la estrategia en materia de seguridad pública iniciada en marzo de 2012 conocida popularmente como La Tregua (Valencia, 13 de abril de 2015). ${ }^{3}$

Aunque este abordaje nunca fue totalmente reconocido por el gobierno anterior, con el acto simbólico que representó una conferencia de prensa brindada después de la reunión, el presidente Sánchez Cerén aceptaba implícitamente el involucramiento del Gobierno, además de dar por concluida esa perspectiva:

Nosotros no podemos volver al esquema de entendernos y de negociar con las pandillas -dijo-, porque eso está al margen de la ley; ellos se han puesto al margen de la ley, ellos se han vuelto violadores de la ley, y por lo tanto nuestra obligación es perseguirlos, castigarlos y que la justicia determine las penas que les corresponden (Valencia, 13 de abril de 2015).

Así el 19 de febrero de 2015, la finalización simbólica de La Tregua se materializó, regresaron

3 Para más información sobre La Tregua, consultar Van der Borg, Chris y Savenije, Wim (28 de abril de 2017). Las lecciones de la Tregua. El Faro, San Salvador. Recuperado de: https://goo.gl/1hRvjq.

4 Para más información sobre los traslados consultar, Valencia, Roberto (14 de marzo de 2015). Zacatraz. El Faro, San Salvador. Recuperado de: https://goo.gl/Nbd5rE.

5 Para más información sobre La Tregua, consultar Van der Borg, Chris y Savenije, Wim (28 de abril de 2017). Las lecciones de la Tregua. E1 Faro, San Salvador. Recuperado de: https://goo.gl/1hRvjq.
14 miembros de la dirigencia, conocidos en el leguaje pandilleril como "palabreros", de la Mara Salvatrucha (MS-13) y las dos facciones del Barrio 18 - Revolucionarios y Sureños -, principales pandillas en El Salvador, para el Centro Penal de Alta Seguridad de Zacatecoluca -llamado popularmente como Zacatraz -, y que era parte central de los acuerdos del proceso impulsado en la administración anterior (Valencia, 13 de abril de 2015). ${ }^{4}$

Después de aproximadamente un mes, comenzaron los síntomas de la anomalía estadística, la cual tendría la capacidad de marcar la historia reciente de El Salvador sobre seguridad pública. En el mes de marzo de 2015, la PNC registró 481 homicidios, con una cifra promedio de 15.52 homicidios intencionales al día (Editorial El Faro, 5 de abril de 2015).

Llegando a consolidarse como el mes más violento en la historia reciente en $\mathrm{El}$ Salvador -entendiendo historia reciente como el siglo XXI-, marzo de 2015 sobrepasó al mes de octubre de 2009 que hasta ese momento tenía el récord con 438 homicidios intencionales. Según los datos consolidados por la PNC y el IML, la cifra de 400 homicidios intencionales al mes solo había sido superada seis veces en la historia del país (Editorial El Faro, 5 de abril de 2015).

Esta situación solo empeoró conforme el tiempo transcurría, siendo agosto el mes con mayor tasa de homicidios registrados, ahora no más en la historia reciente, sino desde la guerra civil salvadoreña - de 1980 hasta 1992 -, llegando a una cifra de 907 homicidios, lo que equivale a 29.25 homicidios intencionales por día (Editorial BBC Mundo, 2 de septiembre de 2015). ${ }^{5}$ 
En este punto, se podía identificar el impacto de la violencia en actividades como el nivel de evasión escolar debido a la intimidación de las jóvenes por las pandillas, ${ }^{6}$ así como también en estrategias que tenían como objetivo demostrar el poder acumulado por las organizaciones criminales para hacer regresar al Gobierno a la mesa de negociación como fue la paralización nacional del transporte público en el mes de julio. ${ }^{7}$

\section{La ineficiencia judicial de los tribunales}

Dentro del campo de la sociología existe una tradición epistemológica llamada la teoría del conflicto, ella parte de la premisa que si todas las interacciones entre los hombres, como Simmel, son formas de asociación, el conflicto como interacción que difícilmente sucedería de forma aislada o sin interlocutor, debería de ser considerado como una forma de relacionamiento (1964, p. 268).

Las sociedades tal y como las conocemos no resultan apenas de fuerzas sociales que les son positivas, sino por el resultado tanto de categorías positivas como negativas -para el statu quo-, que se manifiestan enteramente como desarrolladoras de nuevas realidades (Simmel, 1964, p. 271).

Una de las marcadas diferencias con los teoristas del consenso es el entendimiento de toda acción

\footnotetext{
6 Para más información sobre la deserción escolar por inseguridad, consultar Alvarado, Jimmy (24 de agosto de 2015). La deserción escolar por inseguridad se duplicó em los últimos cinco años, El Faro, San Salvador. Recuperado de: https://goo.g1/3Q8GgM.

7 Para más información sobre la paralización nacional del transporte público, consultar Redacción (29 de julio de 2015). El paro que demuestra el poder de las maras en El Salvador. BBC Mundo. Recuperado de: https://goo.gl/gnxfd8.
}

pública como el resultado de la armonización de los intereses de los miembros de la sociedad alrededor de un objetivo común. Pues los teoristas del conflicto expresan que toda acción pública es el resultado de la disputada por el mantenimiento de la posición socioeconómica de unos estratos sobre otros en la sociedad (L. Snyder, 2013, p. 2).

En este contexto de sociedades con intereses heterogéneos un aparataje institucional que refleja esa estratificación, emerge la necesidad de un tercero elemento. Aquel actor que, funcionada como factor neutro, valorando las múltiples reivindicaciones - a veces contradictorias -, eliminando lo que es incompatible entre ellas y llegando a resultados determinados por una concepción particular de justicia (Simmel, 2015, p. 47).

Así nace la figura del tribunal, arbitro no partidario, el cual toma la decisión final entre las partes en conflicto -ya sea en la sociedad o dentro del Estado-, presuponiendo una confianza subjetiva basada en la objetividad del juzgamiento (Simmel, 2015, p. 53).

Existen dos comportamientos judiciales concomitantes en el ámbito salvadoreño actual: el activismo judicial y la ineficiencia judicial. Cuando se habla del primero, la complejidad se asienta en un poder globalmente político que debe ser aplicado a cada caso concreto de forma apolítica; contradicción con la cual el tribunal nació, pues su legitimidad deriva del carácter apolítico de su ejercicio (De Souza Santos, Leitão Marques y Pedroso, 1995, p. 27).

En el segundo comportamiento, el cual es de particular interés para el presente ejercicio, la 
problemática surge cuando la combinación entre cantidad de casos y demandas hacia los tribunales, los escasos recursos y la falta de voluntad genera una brecha entre los casos judicializados y los resueltos.

Generando de esta forma una tipificación de las fuentes de legitimidad de los tribunales. Constituida por la confianza específica, confianza difusa, confianza en la justicia procesal y la confianza en la efectividad (E. Haas, Keijser y Bruinsma, 2014, p. 227).

Los modelos de confianza específica y difusa, utilizados al momento de evaluar el actuar de las organizaciones, son compilados de forma eficaz por el trabajo de E. Haas, Keijser y Bruinsma (2014) de la siguiente forma:

El apoyo específico se refiere al conjunto de actitudes hacia una institución basada en el cumplimiento de las expectativas de políticas y acciones. Un ciudadano puede estar en desacuerdo con el hecho de que una institución no tenga una confianza difusa pero en una situación específica inclusive podría aceptar esa autoridad como tomador de decisión en general (p. 227).

Por otro lado, aunque los modelos de confianza en la justicia procesual y en la efectividad son utilizados en su mayoría al momento de evaluar la actuación de las organizaciones policiales pueden ayudar también en el caso judicial:

Promotores de este plantamiento mantienen que la legalidad de la policía está basada principalmente en percepciones de equidad, en lugar de tratarse de un problema instrumental. De acuerdo con el modelo instrumental, por otra parte, la legitimidad es más desarrollada y mantenida mediante la eficacia de la policía, que es una evaluación de qué tan bien es la actuación de la policía. (E. Haas, Keijser y Bruinsma, 2014, p. 228).

Pero para poder utilizar estas últimas categorías en el campo de los tribunales, se puede emplear la metáfora de la pirámide judicial en la cual se ubicarían en la base todas las acciones que pueden ser judicializadas y en la punta todas las acciones que son efectivamente judicializadas. Creando la necesidad de un estudio entre el punto de partida y la concretización de la acción judicial (De Souza Santos, Leitão Marques y Pedroso, 1995, p. 44).

Esta aproximación teórica se puede utilizar para identificar el rol de los tribunales como parte del sistema de justicia penal de El Salvador, y sus efectos, frente al contexto de alta violencia criminal que presentó en el año 2015.

En este sentido, tomando como base el trabajo del periódico digital El Faro, quien tramitó a través de la Ley de Acceso a la Información Pública (LAIP) todos los casos del año 2015 que la Fiscalía General de la República (FGR) podía llevar a los tribunales, ahora se conoce que para finales de febrero de 2016 solo uno de cada 10 casos había iniciado su proceso para ser judicializados (Rauda Zablah, 18 de marzo de 2016).

Es decir, transcurridos los primeros dos meses de 2016, la FGR solo había podido presentar ante los tribunales 598 de los 6,650 homicidios ocurridos en el año 2015; es decir, menos del 9\% del total de los homicidios 
intencionales acontecidos (Rauda Zablah, 18 de marzo de 2016).

Tomando como ejemplo enero, donde hubo 336 homicidios, para el mes de febrero de $2016-13$ meses después-, solo 59 de esos casos fueron judicializados. No obstante, otros resultados son el porcentaje de esos casos que obtuvieron sentencia condenatoria.

Pues cuando hablamos de cantidades de condenaciones, la cifra se vuelve más grave, así fue retratado por los abogados entrevistados por El Faro durante su investigación:

Lo más grave, si partimos del número de homicidios registrados por el IML -1.020y lo confrontamos con el total de sentencias condenatorias por homicidios consumados -39-, podemos afirmar que existe sólo un $3.8 \%$ de eficiencia en la investigación del delito y juzgamiento del delincuente, añadieron los abogados. En 2015, en esos mismos municipios hubo 897 homicidios y 13 condenas. Es decir, el porcentaje se redujo a $1.45 \%$ (Rauda Zablah, 18 de marzo de 2016).

Aunque con lo anterior se puede formar la idea de que son los funcionarios del Órgano Judicial los culpables, cuando se hace una aproximación más detallada se encuentra una complejidad mayor. La carga de la prueba que representa el problema de la ineficiencia al momento de juzgar delitos, tenemos que relativizarlo con el número excesivo de casos por fiscal, a la corrupción del sistema de justicia penal y al sistema de pruebas actual que se basa de sobremanera en la figura de los testimonios.
Ejemplos de esto se pueden encontrar en la investigación de Oscar Martínez, donde un fiscal explica que tiene alrededor de 500 casos para ser resueltos:

En un sistema judicial que depende de testigos, el Estado apenas puede protegerlos. En los 10 juicios en los que estuve, todos sabíamos quién era el testigo atrás del biombo. De hecho, el fiscal anónimo asegura que la actitud de muchos policías a la hora de que un testigo pide medidas extraordinarias es desmotivarlo: usted no va a estar solo, no va a tener acceso a celular, va a haber criteriados con usted y su familia, va a estar como preso (9 de mayo de 2016).

Se puede encontrar respaldo de estas afirmaciones también en el Reporte País de las Prácticas sobre Derechos Humanos elaborado por el Departamento de Estado de los Estados Unidos. En el documento se inaugura mencionando que los principales problemas que afectaron los Derechos Humanos en El Salvador en el año 2015 fueron los altos niveles de corrupción, debilidad dentro del judicial y las fuerzas de seguridad que contribuyeron a los niveles altos de impunidad, abuso y discriminación (Bureau of Democracy, Human Rights and Labor, 2015, p.1).

Con todo lo anterior, en El Salvador es razonable cuestionar el rol de los tribunales como árbitros imparciales con la capacidad de mediar entre los conflictos de la sociedad. De igual forma, su poca legitimidad al momento de presentarse como una expresión del poder público neutra y 
autoritaria, encargada de resolver los litigios con su idea universal y abstracta de justicia (Foucault, 2017, p. 26).

\section{E1 uso excesivo de la fuerza policial}

Empero cuando el judiciario es puesto en duda, no son los funcionarios de los tribunales los primeros a ser interpelados por la población, son los policías como primera instancia que entra en contacto con los ciudadanos:

La policía es comúnmente vista como la personificación de este monopolio ya que es el rostro visible, más que otras organizaciones en materia de seguridad y son usualmente la primera instancia de justicia con la que los ciudadados tienen contacto. Es comprensible que cuando los ciudadanos recurren a actos ilegítimos en respuesta al crimen, o toman la justicia por sus propias manos, esto sea considerado como una señal de que la confianza en la policía está en juego (E. Haas, Keijser; Bruinsma, 2014, p. 224).

Así en el contexto de alta violencia criminal, de falta de legitimidad por la ineficiencia judicial y la presión directa de la sociedad, la PNC asumió el rol de la instancia que direccionó las contradicciones internas de la población y las cristalizó en una agencia que no siempre fue justa o legal.

Actuación atribuida la mayoría de las ocasiones solo a la PNC por ser la organización que tiene como núcleo duro de su actuar el uso legítimo de la fuerza física y simbólica. Sin embargo, al igual que otras organizaciones, sea de forma implícita o explícita, la sociedad forma o por lo menos encuadra el comportamiento a través de directrices gubernamentales, mecanismos formales de responsabilidad e influencia de principios, valores, normas y paradigmas (L. Snyder, 2013, p. 3).

Instaurándose así una creencia que ciertas formas de dominación o abuso son legítimas o por lo menos necesarias en ambientes con violencia criminal. Esta creencia se cristaliza desde la declaración de guerra contra las pandillas por parte del Ejecutivo -en enero de 2015- a través de los denominados "enfrentamientos".

El periódico digital El Faro -con ayuda de la LAIP-, obtuvo los listados de todas las situaciones que la PNC clasificó como "enfrentamientos" desde enero de 2015 hasta agosto de 2016. Accediendo también al número de heridos y fallecidos en cada bando - policía y militares por un lado y supuestos pandilleros por el otro-, la localización geográfica del hecho, el sexo y edad de las víctimas mortales (Valencia, 3 de octubre de 2016).

Consolidándose una base de datos donde la PNC registró 1,074 "enfrentamientos" armados con supuestos criminales, con un saldo de 693 muertos y 255 heridos. Para analizar esos resultados, El Faro utilizó el índice de letalidad -IL-, herramienta que establece una relación entre adversarios muertos y heridos en los enfrentamientos entre policías y soldados contra criminales, obteniendo un resultado de IL de 2.72 .

Esto significa, según el especialista brasileño en seguridad pública Ignácio Cano lo siguiente: 
En cualquier enfrentamiento armado legítimo, sea policial o militar, es esperable encontrar un mayor número de heridos que de muertos, con lo que el coeficiente debería ser siempre inferior a 1 (Valencia, 3 de octubre de 2016).

Dejando claro que el espíritu del proceder policial y su propia naturaleza está siendo transformado, pasando de ser un cuerpo civil de seguridad pública y prevención de la violencia, a un actor que reproduce la idea que el uso excesivo de la fuerza -incluyendo la letal- es justificada o legítima cuando busca un bien mayor.

Esta estrategia adoptada por la PNC genera un relevante número de quejas sobre posibles violaciones de los Derechos Humanos. Por ejemplo, para el periodo de junio de 2014 hasta mayo de 2015, se interpusieron alrededor de 2,202 quejas en la Procuraduría para la Defensa de los Derechos Humanos -PDDH-, de las cuales el $92 \%$ fueron atribuidas a la policía y militares. Esto lo respaldó el Ombudsman salvadoreño David Morales:

\section{(...) David Morales notó que PDDH está investigando casos de "arbitrariedad, muertes, situaciones que casi rayan en la tortura y posibles ejecuciones en las escenas de enfrentamientos armados entre la policía y supuestos criminales (Bureau of Democracy, Human Rights and Labor, 2015, p. 2).}

No en tanto, el isomorfismo no deriva de la reproducción irreflexiva de los policías, sino de la regularidad proveniente de la aceptación de determinada acción, que justamente en función de ser interpretada como legítima es reproducida por los agentes en la práctica social y se consolida como un padrón institucionalizado (Machado da Silva, Silva da Fonseca y Crubellate, 2005, p. 29).

En ese sentido, la opinión de la población al respecto del actuar policial se vuelve en un elemento relevante a medir. Así a través de la encuesta de opinión pública realizada por la Unidad de Investigación Social de $L a$ Prensa Gráfica-LPG- entre el 19 y 23 de agosto de 2016 -donde participaron 1,200 personas-, se preguntó a los ciudadanos salvadoreños su posición frente al trabajo hecho por parte de la PNC y si alguna vez fueron víctimas de un maltrato físico por parte de la organización policial.

Los resultados fueron los siguientes: 1) el 54\% de los entrevistados respondió que el trabajo hecho por pate de la PNC era bueno o muy bueno; 2) el $21 \%$, que era malo o muy malo. En comparación con el año 2015, las opiniones eran de 52\% y $22 \%$ respectivamente. Por otro lado, 3) el 16\% contestó que alguna vez fueron víctimas directas de alguna clase de maltrato físico o verbal por parte de la PNC y 4) el 20\% que alguna vez fueron ayudados por la organización policial (Segura, 31 de agosto de 2016).

Aunque los números se pueden mostrar contradictorios, cuando son comparados con estudios en Jamaica, Argentina, India y Brasil, todo indica que el uso excesivo de la fuerza por parte de la policía es tolerado y entendido como una forma necesaria de control o coerción social, por eso no se traducen en números negativos, sino por el contrario generan legitimidad a la organización (L. Snyder, 2013, p. 6). 
No obstante, la idea que existen sociedades condenadas a la violencia por el hecho de ellas ser violentas sería también una interpretación limitada la compleja realidad social. Aunque escapa al alcance del presente, vale la pena mencionar que en situaciones de alta violencia -física, simbólica y estructural- se crean comportamientos colectivos que hacen emerger sociedades fraccionarias y temporales distintas a las habituales en situaciones pacificas (Martins, 2015, p. 63).

Donde se posicionan el control y la represión como instituciones principales de la seguridad pública, olvidando el paradigma de la seguridad ciudadana y humana e identificando como desviantes -que tiene que ser combatidos-a los narradores de abordajes diferentes.

Un ejemplo de esto último fue registrado por el Reporte País del Departamento de Estado que ya mencionado anteriormente y que describe lo siguiente:

(...) Reporter Oscar Martinez, from the online journal El Faro, reported on-line harassment, including death threats after publishing a July 2 article that quoted a conversation between PNC officers in which officers encouraged other officers to kill detained gang members. El Faro also reported being subject to online harassment and death threats following its July 22 report alleging a police cover-up of the illegal killing of eight civilians by police at the San Blas Farm on March 26 (Bureau of Democracy, Human Rights and Labor, 2015, p. 11).
Debido a esta situación de uso excesivo de la fuerza y significativas denuncias de violación de los Derechos Humanos, a solicitud del Departamento de Estado, el Director General de la PNC en ese momento estableció lo siguiente:

The Inspectoria General -IG- office collects, complaints and independently investigates allegations of police misconduct. As of September, the newly independent IG reported authorities investigated eight police officers for homicide. In the same period, the IG also received 709 complaints of alleged police misconduct, referred seven of these cases to the FGR, and sanctioned 296 officers in response to complaints filed during the year and in prior years. These sanctions included 62 officers dismissed for misconduct and 234 suspended without pay. As of September 3, the FGR had investigated 234 accusations against police officers, resulting in five cases resolved through mediation and seven convictions (Bureau of Democracy, Human Rights and Labor, 2015, p.7).

Datos que llevan a la hipótesis - debido a la baja taza de casos juzgados frente al número de acusaciones -, con base en los estudios de L. Snyder, que el posible motivo del involucramiento de la organización de control interno de la PNC - IG - solo busca controlar las consecuencias resultantes de la presión pública sobre la organización, esto en lugar de interesarse por las consecuencias tanto internas como externas que pueden generar los hechos cometidos por los policías (2013, p. 4).

Además de lo mencionado anteriormente y sin ánimos de minimizarlo, este entorno es propicio también cuando en los sistemas liberales, 
los estratos socioeconómicos altos y medios dejan de servir como factores de moderación. Adoptando la idea de la represión como la solución al problema de la violencia criminal y reproduciéndola en toda la sociedad al poseer suficiente capital social y cultural que la legitime. Pues como menciona Foucault, la verdad no existe fuera del poder o sin el poder, cada sociedad escoge y hace funcionar discursos que permiten distinguir los enunciados verdaderos de los falsos y la manera como se sancionan unos y a los otros; las técnicas y los procedimientos que son valorizados para obtener la verdad; y el estatuto de aquellos que tiene el encargo de decir lo que funciona como verdadero (2017, p. 10).

\section{Las pandillas como organizaciones terroristas}

Un ejemplo claro del peso que los discursos de las organizaciones creadoras de la verdad tienen en el área de la seguridad pública sucedió el día 24 de agosto de 2015. Cuando la Sala de lo Constitucional de la Corte Suprema de Justicia -SC/CSJ-, a través de un proceso de inconstitucionalidad acumulado, resolvió 4 demandas interpuestas en el año 2007 contra la Ley Especial contra Actos Terroristas-LECAT-, sentencia en la cual rechazan 23 argumentos y aceptan 4 elementos inconstitucionales establecidos en la ley (Sala de lo Constitucional, 24 de agosto de 2015).

Aunque puede parecer contradictorio, en este caso en particular la importancia de la sentencia se encuentra en los elementos ponderados como constitucionales y no en los elementos expulsados del ordenamiento jurídico salvadoreño. Así en la ratio decidendi -parte de la sentencia que se encarga de desarrollar la argumentación sobre la cual se tomó la decisión final- la SC/CSJ expresa:

La Sala concluye que son grupos terroristas las pandillas denominadas Mara Salvatrucha o MS-13 y la Pandilla 18 o mara 18, y cualquier otra pandilla $u$ organización criminal que busque arrogarse el ejercicio de las potestades pertenecientes al ámbito de la soberaníadelEstado, atemorizando, poniendo en grave riesgo o afectando sistemática e indiscriminadamente los derechos fundamentales de la población o de parte de ella; en consecuencia, sus jefes, miembros, colaboradores, apologistas y financistas, quedan comprendidos dentro del concepto de "terroristas" en sus diferentes grupos y formas de participación, e independientemente de que tales grupos armados $\mathrm{u}$ organizaciones delictivas tengan fines políticos, criminales, económicos (extorsiones, lavado de dinero, narcotráfico, etc.), o de otra índole (Sala de lo Constitucional, 24 de agosto de 2015).

Atribuyendo de esa forma la tipificación de terroristas a las principales pandillas de El Salvador, y cualquier otra organización criminal que tenga una naturaleza similar en el futuro, dividiendo la opinión sobre los futuros efectos de esta decisión. En primer lugar, tenemos la interpretación de los representantes del Ejecutivo, como en ese momento el subdirector de la PNC Howard Cotto que menciona, "lo que implica es perseguir a estos grupos criminales por su condición y no solo por los actos que hayan cometido" (Del Cid, 25 de agosto de 2015).

En segundo lugar, la interpretación del tanque de pensamiento salvadoreño más influyente, 
la Fundación Salvadoreña para el Desarrollo Económico y Social (FUSADES) que identifica como único efecto inmediato la necesidad de juzgar conforme a la LECAT como parte de organizaciones terroristas $^{8}$ a los miembros de las pandillas, y no más conforme al delito de agrupaciones ilícitas. ${ }^{9}$ Cualquier otro hecho para ser clasificado como terrorista debería constar en la ley y declararse así en juzgamiento de cada caso en particular (Departamento de Estudios Legales, 2015).

En tercer lugar, la interpretación más crítica, la cual proviene de las organizaciones de la sociedad civil y se resume en dos ideas clave: 1) además del potencial abuso de autoridad que puede generar, esta decisión puede también afectar el trabajo que realizan organizaciones sin fines de lucro y organismos internacionales con jóvenes en riesgo que viven dentro de áreas controladas por las pandillas o el trabajo realizado para ayudar a miembros activos a dejar sus vidas criminales (Daugherty, 27 agosto 2015).

Y 2) la priorización en el sistema jurídico salvadoreño del derecho penal del enemigo, el cual tiene como principales características el uso de la punición como una de las primeras acciones, la desproporcionalidad de la pena versus el acto y la supresión de algunas garantías constitucionales dentro del proceso de juzgamiento (Landaverde, 26 de agosto de 2015).

De igual forma, resulta claro en la sentencia la

8 Reconocido en el artículo 13 de la LECAT de 2006 y vigente hasta la fecha de publicación del presente, estableciendo penas de 8 a 15 años de prisión.

9 Reconocido en el artículo 345 del Código Penal salvadoreño de 1973 y vigente hasta la fecha de publicación del presente, estableciendo penas de 3 a 9 años de prisión. imposibilidad del establecimiento de canales de comunicación con las organizaciones criminales denominas como terroristas, mediante el establecimiento de lo siguiente:

No resulta aceptable dentro del marco del respeto de la Constitución y la ley, la formulación de acuerdos de no persecución criminal o el establecimiento de alguna prerrogativa para dispensar la aplicación de las disposiciones jurídicas para quien las viole, pues ello pone en entredicho el mismo ordenamiento jurídico y el ejercicio de fidelidad al derecho que todo funcionario y ciudadano debe tener en relación con este último (Rauda Zablah, 25 de agosto de 2015).

Fue en este contexto que el año 2015 terminó con una cifra de aproximadamente 6,650 homicidios, equivaliendo a un $\mathrm{IH}$ de 103 para cada 100,000 habitantes. Para tener una idea sobre el significado de esta cifra, El Salvador tiene el $13.6 \%$ de la población en América Latina; sin embargo, concentra el $35.3 \%$ de los homicidios en la región. En otras palabras, uno de cada tres homicidios en 2015 en América Latina fue cometido en el país (Segura, 3 de enero de 2016).

A nivel regional, el promedio del IH fue de 40.2 para cada 100,000 para el año 2015, superada fácilmente por El Salvador, viéndose así como el país más violento en la zona más violenta del mundo sin conflicto armado. Dejando en segundo y tercero lugar a los países de Venezuela y Honduras con un promedio de IH de 90 y 57 para cada 100,000 , respectivamente. 
Finalizando, existen escasas evidencias que demuestren que el abordaje puramente represivo en seguridad pública sirve para reducir la violencia criminal, lo que puede significar, en algunas ocasiones, gestionar la violencia con más violencia, creando un círculo vicioso. Sin embargo, esta fue la perspectiva decidida por el Ejecutivo en enero de 2015, operacionalizada por la PNC, agravada por los tribunales y respaldada por la SC/CSJ.

\section{Consideraciones finales}

El año 2015 en El Salvador fue inaugurado con un fuerte cambio del abordaje en materia de seguridad pública por parte del nuevo Gobierno -tenía 7 meses de haber iniciado su gestión. Dejando claro que la estrategia de negociación o mediación entre y con las pandillas- hasta la fecha de publicación del presente La Tregua sigue sin transparentarse no sería más la forma de gestionar la violencia criminal, y en lugar de eso se aplicaría una estrategia de confrontación directa contra las organizaciones criminales.

Después de alrededor de un mes, los resultados de esta nueva perspectiva se estaban presentando en la forma de aumento de los homicidios intencionales a niveles alarmantes. Esto pudo ser interpretado como una manera de presión por parte de las organizaciones criminales para hacer volver al Ejecutivo a la estrategia del anterior Gobierno; como una demostración que las pandillas habían incrementado su capacidad de agencia durante el periodo de La Tregua; o como la eficacia de las medidas implementadas al crear una reacción por parte de las pandillas frente al debilitamiento de sus estructuras organizacionales.
Al margen de las diferentes lecturas sobre estas circunstancias, el hecho fue que con el aumento del número de homicidios también crecieron el número de enfrentamiento entre la PNC y supuestos miembros de las pandillas, o viceversa, con el aumento de enfrentamiento creció el número de homicidios. Dejando al ya débil sistema judicial en problema al tener que resolver una cantidad de casos sin los recursos necesarios - en el sentido lato de la palabra.

Frente a esta ineficiencia judicial, la estrategia demarcada por el Ejecutivo y la posible presión de las pandillas, se delinearon las condiciones propensas para el uso excesivo de la fuerza por parte de la PNC como herramienta de materialización del objetivo de la organización que es mantener el orden público. Vale aclarar que la utilización del concepto fuerza cuando se refiere a las organizaciones públicas y violencia cuando se refiere a las organizaciones criminales fue utilizado para diferenciar la legalidad o ilegalidad de su naturaleza.

De igual forma, al momento de utilizar la expresión uso excesivo de la fuerza se reconoce su naturaleza ilegal, teniendo en mente que su génesis ocurrió dentro de la legalidad vigente excluyendo el factor de la legitimidad de las acciones porque ellas pueden tener tanto fuentes legales como ilegales de apoyo.

Transformando a los policías que cometieron delitos en los portadores del significado institucional del uso legítimo de la fuerza excesiva. Infundiendo actitudes dentro de las organizaciones por medio de la interpretación de factores internos y externo de apoyo y oposición, y corriendo el riesgo de ser legitimados por 
su socialización (Machado da Silva, Silva da Fonseca y Crubellate, 2005, p. 28).

Identificando a estos actores policiales como los emprendedores institucionales que buscan -de forma implícita o explícita- la adopción como viable, conveniente y deseable de la gestión represiva de la violencia criminal. Teniendo el potencial de generar un isomorfismo mimético dentro de la organización policial sino se acentúa una posición firme en contra de esta corriente.

Pasando, a través del presente, la pregunta más frecuente al momento de los estudios del ejercicio de la fuerza policial como dice Brodeur, la cual es la interrogante del problema fáctico, responder sobre la extensión y características del uso excesivo de la fuerza; llegando a la duda de naturaleza normativa, teniendo como objetivo indagar la relación entre la cultura organizacional y societaria y el uso excesivo de la fuerza por parte de los policías (2003, p. 204).

Constatando a través de las encuestas de opinión pública hechas por LPG, que la estrategia de gestión represiva operacionalizada por la PNC ser convirtió en una fuente de legitimidad para la organización. Creando un efecto espejo de las conductas criminales por parte de las organizaciones que quieren compartirlas (Kowalewski, 2003, p. 343).

Abriendo peligrosamente el escenario para el fenómeno del vigilantismo y sus contramovimientos que solo causarían más violencia. Legitimando actitudes violentas en lugar de transformarlas, disminuyendo la poca confianza que todavía existe en el sistema de justicia penal y duplicando errores históricos sucedidos durante el periodo de la guerra civil salvadoreña (1980/1992).

\section{Referencias bibliográficas}

Bureau of Democracy, Human Rights and Labor (2015). El Salvador Human Rights Report. Country reports for Human Rights practices. United States Department of State. Washington.

Brodeur,J. (2003).Violence and police. En: Heitmeyer, Wilhelm y Hagan, John (Ed.). International Handbook of Violence Research. New York: Klumer Academic Publishers, p. 207-224.

Daugherty, A. (27 de agosto de 2015). Corte Suprema de Justicia de El Salvador declara como grupos terroristas a las pandillas. InSightCrime. Recuperado de: Https://goo.gl/nbfLJx.

Del Cid, M. (25 de agosto de 2015). El Salvador declara como grupos terroristas a las pandillas. $C N N$ Español. Recuperado de: https://goo.gl/ztT5LY.

De Souza Santos, B., Leitão Marques, M. y Pedroso, J. (1995). Os tribunais nas sociedades contemporâneas. Universidade de Coimbra. Centro de Estudos Sociais, Oficina de Estudos Sociais.

Departamento de Estudios Legales (2015). Administración de justicia. En: FUSADES. Informe de coyuntura legal e institucional. Antiguo Cuscatlán: El Salvador. Recuperado de: https://goo.gl/6HXph7.

ABC Internacional. (2 de septiembre de 2015). ¿Por qué El Salvador considera ahora a las maras como terroristas? $A B C$ Internacional, Madrid. Recuperado de: Https://goo.gl/HKNHyT. 
BBC Mundo (2 de septiembre de 2015). El Salvador confirma a agosto como el mes más violento desde la guerra civil. $B B C$ Mundo. Recuperado de: https://goo.gl/4CX9Q6.

Editorial El Faro (5 de abril de 2015). Con 481 asesinatos, marzo se convirtió en el mes más violento del siglo. El Faro, San Salvador. Recuperado de: https://goo.gl/aCVXB7.

Editorial El Faro (3 de octubre de 2016). Los datos apuntas a la presencia de ejecuciones sumarias. El Faro, San Salvador. Recuperado de: https://goo.g1/J3K7UB.

E. Haas, N., Keijser, J. y Bruinsma, G. (2014). Public support for vigilantism, confidence in police and police responsiveness. Policing and Society: An International Journal of Research and Policy, Vol. 24, No. 2, p. 224-241.

Foucault,M.(Ed.5a) (2017). A microfísica do poder. Organización y traducción por Roberto Machado. Rio de Janeiro: Ediciones Paz \& Tierra.

Gagne, D. (15 de enero de 2016). Balance de InSight Crime sobrehomicidios emLatinoamérica en 2015. InSight Crime, Washington. Recuperado de: Https://goo.g1/tciYqn.

Kowalewski, David (2003). Vigiliantism. En: Heitmeyer W. y Hagan, J. (Ed.). International Handbook of Violence Research. Netherland: Kluwer Academic Publishers: p. 339-349.

Machado da Silva, C.L., Silva da Fonseca, V. y Crubellate, J. M. (2005). Estrutura, Agência e Interpretação: Elementos para uma Abordagem Recursiva do Processo de Institucionalização.
Brazilian Administration Review, v. 2, n. 1, enero-junio, p. 09-39.

Martínez, O. (11 de febrero de 2016). La policía mata y miente de nuevo. El Faro, San Salvador. Recuperado de: https://goo.g1/z9Z1vD.

Martínez, O. (9 de mayo de 2016). El Salvador es un buen lugar para matar. El Faro, San Salvador. Recuperado de: https://goo.g1/z5YD8x.

Martins, J. (2015). Linchamentos: justiça popular no Brasil. São Paulo: Contexto.

Mendoza, B. (24 de agosto de 2015). Pandillas declaradas como grupos terroristas por la Sala. La Prensa Gráfica, San Salvador. Recuperado de: Https://goo.g1/Kj5PvJ.

Najar, A. (25 de agosto de 2015). ¿Qué significa que declaren terroristas a las maras en E1 Salvador? BBC Mundo, Madrid. Recuperado de: https://goo.gl/Xm96EX.

Landaverde, M. (8 de septiembre de 2015). La declaratoria de terroristas a las pandillas criminales hecho por la Sala de los Constitucional. Revista Digital Enfoque Jurídico, San Salvador. Recuperado de: https://goo.gl/k18FkW.

Landaverde, M. (26 de agosto de 2015). E1 derecho penal del enemigo. Revista Digital Enfoque Jurídico, San Salvador. Recuperado de: Https://goo.gl/ZBGqQI.

L. Snyder, B. (2013). Policing the police: Conflict theory and police violence in a racialized society. A thesis submitted in partial fulfillment of the requirements for the degree of Master of Arts. 
Washington D.C: Department of Sociology, University of Washington.

L. Deephouse, D. y Suchman, M. (2015). Legitimacy in organizational institutionalism. En: Greenwood, Royston (Ed.). The SAGE Handbook Organizational Institutionalism. SAGE Publications.

Programa de las Naciones Unidas para el Desarrollo. (2013). Seguridad Ciudadana con Rostro Humano: diagnóstico y propuestas para América Latina. Informe Regional de Desarrollo Humano 2013-2014. Nueva York: Autor.

Rauda Zablah, N. (18 de marzo de 2016). La fiscalía solo ha podido llevar a tribunales uno de cada 10 homicidios cometidos en 2015. E1 Faro, San Salvador. Recuperado de: https://goo.g1/ EBNBsN.

Rauda Zablah, N. (25 de agosto de 2015). Sala de lo Constitucional declara ilegal negociación con pandillas y las nombra grupos terroristas. El Faro, San Salvador. Recuperado de: https://goo. $\mathrm{g} 1 / 6 \mathrm{~N} 9 \mathrm{Nj}$.

Sala de lo Constitucional (24 de agosto de 2015). Procesos de inconstitucionalidad 22-20007; 422007; 89-2007 y 96-2007. San Salvador: Corte Suprema de Justicia.

Segura, E. (3 de enero de 2016). El Salvador con más homicidios en Centroamérica. La Prensa Gráfica, San Salvador. Recuperado de: https:// goo.g1/2vXbLC.

Segura, Edwin (31 de agosto de 2016). Mayoría de salvadoreños con opinión positiva sobre la PNC. La Prensa Gráfica, San Salvador. Recuperado de: https://goo.gl/k7yzTD.

Simmel, George (2015). Sociología: Estudios Sobre las Formas de Socialización. Fondo de Cultura Económica. México.

Simmel, Georg (1964), O conflito como associação. Tradução de Mauro Guilherme Pinheiro Koury. Revista Brasileira de Sociologia da Emoção, v. 10, n. 30, p. 568-573.

Unidad Investigativa sobre Venezuela (17 de enero de 2016), Balance de InSight Crime sobre homicidios en Latinoamérica en 2016. InSight Crime, Washington. Recuperado de: https://goo. gl/JFifr8.

The Associated Press (3 de enero de 2016). La policía de E1 Salvador dice que en 2016 se redujeron los asesinatos en el país. The Nerw York Times, New York. Recuperado de: https://goo.gl/ tURvXe.

Valencia, R. (13 de abril de 2015). El obituario de la Tregua. El Faro, San Salvador. Recuperado de: https://goo.g1/PXTZv2.

Valencia, Roberto (13 de abril de 2016). "Mano dura es la de ahora, no la de Paco Flores”. E1 Faro, San Salvador. Recuperado de: https://goo. $\mathrm{gl} / \mathrm{gF} 39 \mathrm{bi}$.

Valencia, R. (1 de junio de 2016). Mayo cierra con el promedio de asesinatos más bajo de los últimos 15 meses. El Faro, San Salvador. Recuperado de: https://goo.gl/wa7Sn3.

Valencia, R. (5 de julio de 2016). Las pandillas 
caminan frente a un frente común ante medidas extraordinarias. El Faro, San Salvador. Recuperado de: https://goo.gl/uArgC3.

Valencia, R. (19 de agosto de 2015). La tasa de homicidios de El Salvador supera a la de Honduras. El Faro, San Salvador. Recuperado de: https://goo.gl/gpCQLF.

Valencia, R. (3 de octubre de 2016). Casi que guardia nacional. El Faro, San Salvador. Recuperado de: https://goo.gl/C3UPf9.
Valencia, R. y Martínez, C. (10 de enero de 2016). "Todo nos indica que las autoridades están cometiendo asesinatos extrajudiciarios". $E l$ Faro, San Salvador. Recuperado de: https://goo. g1/MLUjQK.

Valencia, R.; Martínez, C.(22 de enero de 2016). La policía masacró en la finca de San Blas... Y todo apunta a que triunfará la impunidad. El Faro, San Salvador. Recuperado de: https://goo. $\mathrm{g} 1 / \mathrm{EwmCMg}$. 